\title{
Analytical Performance of a New Gliadin-Activated Anti-tTransglut- aminase ELISA Test
}

\author{
Xavier Galan*, ${ }^{*}$, Marcos López Hoyos $^{2}$, Eva Cacho ${ }^{2}$, Isabel Goirigolzarri ${ }^{2}$ and Petraki Munujos ${ }^{1}$ \\ ${ }^{1}$ Research and Development Department, BioSystems S.A., Barcelona, Catalonia, Spain \\ ${ }^{2}$ Servicio de Inmunología, Hospital Universitario Marqués de Valdecilla, Santander, Spain
}

\begin{abstract}
Significant progresses on the anti-tissue transglutaminase antibodies (anti-tTG) ELISA tests have been achieved since anti-tTG early development. Our goal was to study the optimization of the antigen immobilization and the antibody reaction conditions in an ELISA test for the detection of anti-tTG. Hence, three variants of an anti-tTG ELISA test were developed: tTG-Gliad-U, tTG-U and tTG-Gliad, according to the coating conditions (presence/absence of gliadin) and the stringency in the sample buffer (presence/absence of urea). To assess IgA and IgG anti-tTG frequencies we used a group of $117 \mathrm{CD}$ patients, 36 under a gluten-free diet and 14 with a selective IgA deficiency, and a control group of 165 non-celiac patients. Sensitivity of IgA anti-tTG in CD patients with villous atrophy was $95.5 \%$ (tTG-Gliad-U), $65.0 \%$ (tTG-U) and 92.5\% (tTG-Gliad), respectively. Sensitivity of the IgG anti-tTG assay was 79.0\%, $21.0 \%$ and 79.0\%, respectively. Specificities ranged between $92.9 \%$ and $100 \%$. Significant lower IgA and IgG concentration values were observed in GFD CD patients. Sensitivity and specificity of IgG anti-tTG in CD patients with selective IgA deficiency were 64.3 and $100 \%$, respectively. In conclusion, we have developed an ELISA test for IgA anti-tTG based on calcium and gliadin activated recombinant human transglutaminase. This test is sensitive and specific, and it is useful to ensure the compliance with diet of CD patients.
\end{abstract}

Keywords: Tissue transglutaminase, ELISA, Coeliac disease.

\section{INTRODUCTION}

Anti-tissue transglutaminase antibodies (anti-tTG) have become the most relevant serological markers for the diagnosis of celiac disease (CD) [1]. Since the description of their utility in $\mathrm{CD}$, many technical improvements have been introduced in the ELISA tests, and significant differences have been described, basically on sensitivity, depending on the experimental setting of the assay, special coating conditions and antigen characteristics.

Tissue transglutaminase (tTG) is a calcium-dependent enzyme that cross-links proteins by a two-step reaction, catalyzing the formation of a covalent bond between proteinbound glutamines (first step) and lysines (second step) [2,3]. Among the different cereal proteins, gliadin, which is rich in glutamine and proline, is a good substrate for tTG, converting glutamine residues to glutamic acid by deamidation. It is well known that $\mathrm{Ca}^{2+}$ plays an important role in the crosslink formation between the tTG and the gliadin during the enzymatic reaction. tTG can cross-link glutamine residues of peptides to lysine residues in TTG itself, which may result in the formation of complexes of gluten-tTG leading to the idea of gluten-dependence of tTG autoantibodies [4]. tTG-mediated reactions with polyamines, such as gliadin, may result in protein modification possibly affecting their antigenicity [5].

Activation of tTG by gliadin in the presence of calcium may play a role for a complete expression of tTG autoepi-

*Address correspondence to this author at the Research and Development Department, BioSystems S.A., Barcelona, Catalonia, Spain; Tel: +34-933110000; Fax: +34-933115497; E-mail: xgalan@biosystems.es topes during the coating process [2-5]. Also the stringency of the sample diluent can contribute to increase specificity of the interaction antibody-antigen.

Our aim was to optimize the antigen immobilization and the antibody reaction conditions in an anti-tTG ELISA and to do so we have compared the performance of three antitTG ELISA test variants, combining the presence or absence of gliadin in the coating mixture together with the use of urea in the sample diluent. Evaluation of the significance of IgG anti-tTG in CD diagnosis was also considered in these studies.

\section{MATERIALS AND METHODOLOGY}

\section{Patients and Sera}

Sera from $117 \mathrm{CD}$ patients (29 males, 88 females, mean age 16, standard deviation 20) were used in different experiments. To calculate the diagnostic characteristics of antitissue transglutaminase ELISA test, a group of 67 sera were selected according to the following criteria: $\mathrm{CD}$ was confirmed by total or subtotal villous atrophy, they were not receiving any treatment at the moment of the serum extraction and had not followed any special diet. A second group of 36 sera came from CD patients under a gluten-free diet (GFD) and samples were taken before and after the treatment, and a third group from $14 \mathrm{CD}$ patients with a selective $\operatorname{IgA}$ deficiency in order to evaluate the usefulness of $\operatorname{IgG}$ anti-tTG in such patients. 165 sera of non-celiac patients were used as control groups: 66 from Crohn's disease (26 males, 40 females, mean age 35, standard deviation 15), 72 from type-1 Diabetes Mellitus (28 males, 44 females, mean age 18, standard deviation 22), 12 from Down syndrome (5 
males, 7 females, mean age 19, standard deviation 8) and 15 from non-CD IgA deficiency (5 males, 10 females, mean age 19, standard deviation 20).

Sera were collected from the routine samples consecutively submitted to the Immunology laboratory, and stored at $-80^{\circ} \mathrm{C}$ until use. Sample selection was done by technicians unaware of the aim of the study. Diagnosis was confirmed by Dr. Marcos López-Hoyos after complete review of clinical records. Celiac and Crohn diseases were confirmed by biopsy. Samples were collected in agreement with the Ethical guidelines of the Hospital Universitario Marqués de Valdecilla (Santander, Spain) and the 2007 Spanish law for biobanks.

\section{Anti-Tissue Transglutaminase ELISA}

Three variants of an ELISA test for the detection of $\mathrm{IgA} / \mathrm{IgG}$ anti-tissue transglutaminase (tTG) antibodies were developed using different antigen treatments and reaction mixtures. Briefly, polystyrene 96 well microplates (Maxisorp Nunc, Roskilde, Denmark) were coated with a $1 \mu \mathrm{g} / \mathrm{mL}$ solution of human recombinant tTG (Diarect, Freiburg, Germany), previously subjected to gliadin activation with gliadin (tTG-Gliad) or not (tTG). In both cases the composition of the coating buffer was $25 \mathrm{mmol} / \mathrm{L}$ Tris, $10 \mathrm{mmol} / \mathrm{L} \mathrm{CaCl}_{2}$

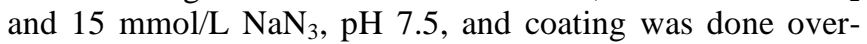
night at $2-10^{\circ} \mathrm{C}$, followed by 3 washes of a blocking solution containing $20 \mathrm{~g} / \mathrm{L}$ bovine serum albumin and $0.5 \mathrm{~mol} / \mathrm{L}$ sucrose. All microplates were dried out for $1 \mathrm{~h}$ at $37^{\circ} \mathrm{C}$, sealed in an anhydrous atmosphere and stored at $2-10^{\circ} \mathrm{C}$ until use. In the sample buffer another variant was incorporated with the presence or absence of urea (U). According to the combination of coating conditions and stringency in the sample buffer, the three variants of the ELISA test were: tTG-Gliad-U, tTG-U and tTG-Gliad. All samples were diluted $1 / 100$ and incubated for 30 minutes at room temperature. The conjugates used were horseradish peroxidase antihuman IgA and horseradish peroxidase anti-human IgG (Dako, Glostrup, Denmark) and incubation time was $30 \mathrm{~min}$ utes at room temperature. 3,3',5,5'-Tetramethyl benzidine (Kementec Diagnostics-Immunoreagents, Copenhagen, Denmark) was used as the horseradish peroxidase substrate.

tTG-Gliad-U

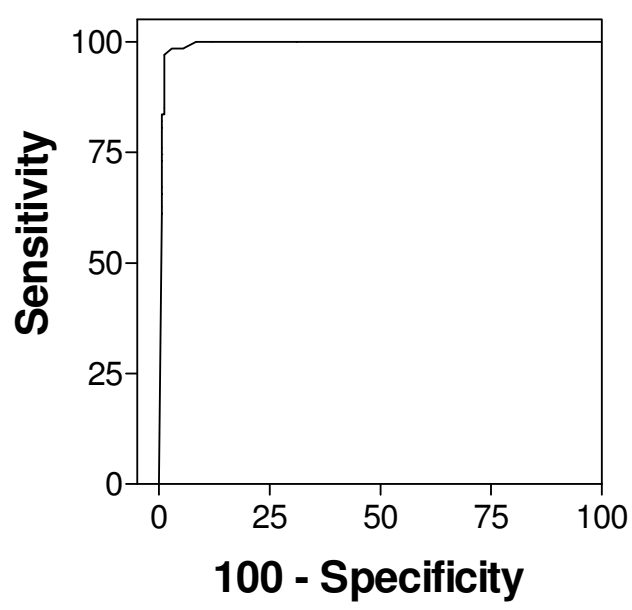

tTG-U

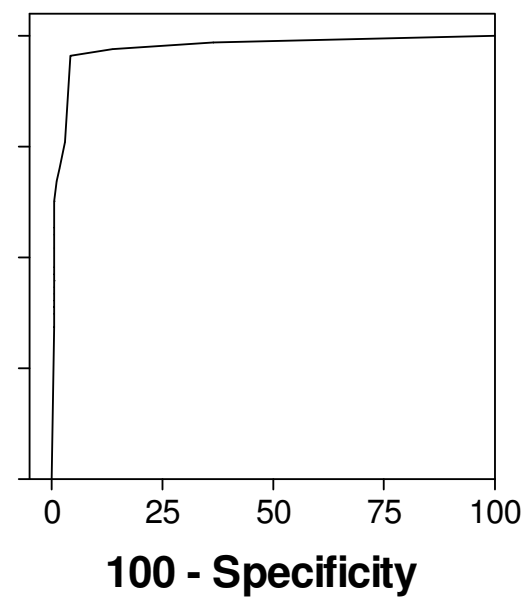

substrate. After 15 minutes of incubation at room temperature, the enzymatic reaction was stopped with $0.5 \mathrm{M} \mathrm{H}_{2} \mathrm{SO}_{4}$, and absorbance was read at $450 \mathrm{~nm}$. ries.

One replicate of each sample was run in at least two se-

\section{Statistical Analysis}

ROC curve analysis was performed with the SPSS statistical package (release 15.0 software, Chicago, IL) and it was carried out for each condition considering as disease group the $\mathrm{CD}$ patients and as non-disease group all the non-CD patients indicated above.

The significance of differences before and after GFD was tested using the Student $t$-test for paired samples. $P$ values lower than 0.05 were considered statistically significant.

\section{RESULTS}

\section{Cut-off Determination}

Samples from 67 untreated CD patients with total or subtotal villous atrophy, and samples from 165 non-CD patients were used to set up the discrimination value for the three anti-tTG antibodies ELISA variants developed for both, IgA and IgG isotypes: tTG-U, tTG-Gliad and tTG-Gliad-U. The absorbances obtained were transformed to arbitrary units $(\mathrm{U} / \mathrm{mL})$ using dilutions of a serum with a high antibody concentration and considered as an internal standard set up in preliminary studies (data not shown). The calibration curve generated in each assay was fitted to a non-linear regression model described by the equation of a rectangular hyperbola.

For each condition, a ROC analysis was made to establish a cut-off value (Fig. 1). The area under the ROC curve using the IgA conjugate was 0.994 (95\% CI, 0.987-1.001) for the tTG-U condition; 0.965 (95\% CI, 0.931-1.000) for the tTG-Gliad condition; and 0.945 (95\% CI, 0.899-0.990) for the tTG-Gliad-U. The best discrimination between patients with active celiac disease and the control group was achieved by a selected cut-off value of $10 \mathrm{U} / \mathrm{mL}$ (arbitrary units) for the three variants with the anti-IgA conjugate. The area under the ROC curve using the IgG conjugate for the 
tTG-IgA

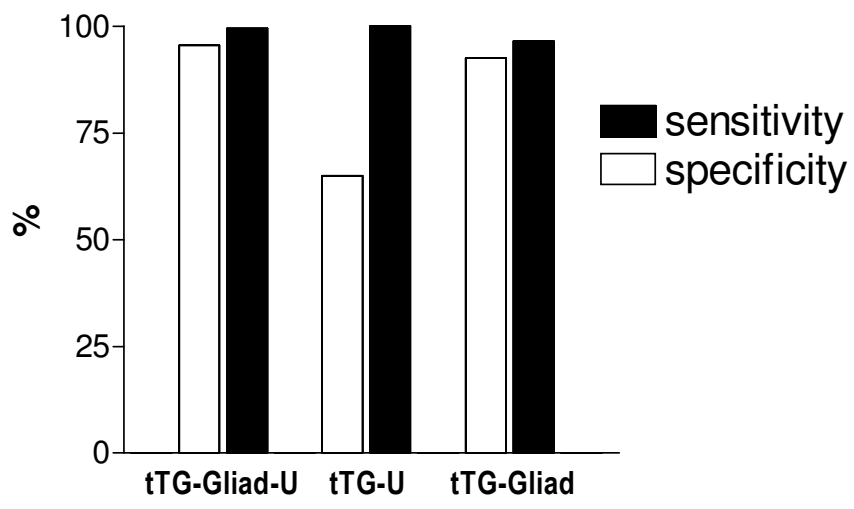

tTG-IgG

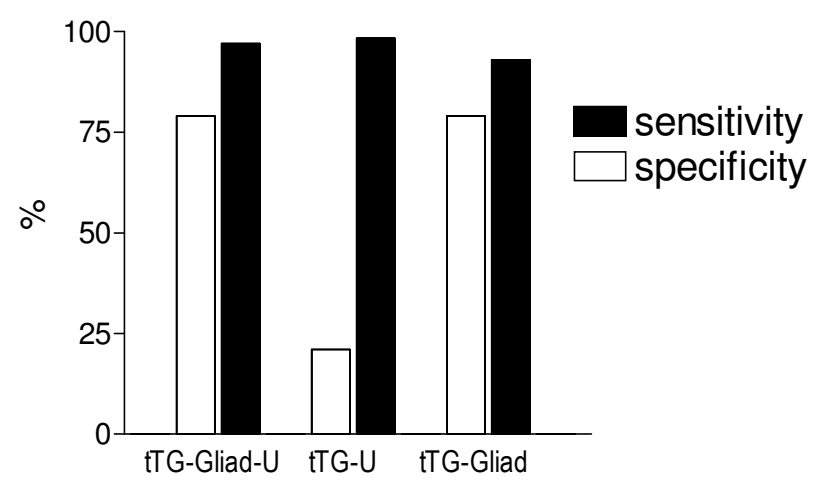

Fig. (2). Diagnostic sensitivity and specificity of the tests. Detection of anti-tTG-IgA and IgG-tTG antibodies in 67 untreated celiac patients showing total or subtotal villous atrophy and 165 non-celiac patients, using the three ELISA variants.

tTG-U, tTG-Gliad and tTG-Gliad-U condition was, respectively, 0.979 (95\% CI, 0.963-0.994), 0.804 (95\% CI, 0.7240.884 ) and 0.856 (95\% CI, 0.796-0.915). Using the anti-IgG conjugate the cut-off was fixed at $5 \mathrm{U} / \mathrm{mL}$ for the three variants.

\section{Diagnostic Sensitivity and Specificity}

IgA anti-tTG sensitivity and specificity were calculated using the selected cut-off value. Sensitivity was $95.5 \%$ for tTG-Gliad-U, $65.0 \%$ for tTG-U and $92.5 \%$ for tTG-Gliad (Fig. 2), and specificity was $99.4 \%$ for tTG-Gliad-U, $100.0 \%$ for tTG-U and $96.4 \%$ for tTG-Gliad (Fig. 2, Table 1). The tTG-Gliad is the sole condition where false positives are found in Type I diabetes patients (Table 1).

IgG anti-tTG sensitivity was $79.0 \%$ for tTG-Gliad-U, $21.0 \%$ for $\mathrm{tTG}-\mathrm{U}$ and $79.0 \%$ for tTG-Gliad, whereas specificity was $97.0 \%$ for tTG-Gliad-U, $98.2 \%$ for tTG-U and 92.9\% for tTG-Gliad (Fig. 2).

\section{Serum Antibodies During Gluten Free Diet Period}

Levels of IgA anti-tTG antibodies before and after introducing a GFD were measured in 36 celiac patients confirmed by biopsy (total, subtotal or partial) using the three ELISA variants developed (Fig. 3). For the tTG-Gliad-U condition, 36 out of 36 samples were positive before introducing the
GFD (mean $198.0 \pm 81.0 \mathrm{U} / \mathrm{mL}$ ). During the follow up, 26 out of 36 samples became negative with concentrations below the cut-off value $(10 \mathrm{U} / \mathrm{mL}) .10$ samples remained positive. One of them (with a partial villous atrophy) did not change after the GFD was introduced (188 U/mL before; 179 $\mathrm{U} / \mathrm{mL}$ after GFD), but concentration of the other 9 samples was reduced between 4 and 25 times (mean $31.0 \pm 21.9$ $\mathrm{U} / \mathrm{mL}$ ). For the tTG-U condition, only 25 out of 36 samples were positive before introducing the GFD. After the retrieval of gliadin from the diet, 17 out of 25 samples were negative and 8 samples remained positive. One of them (the same as in the tTG-Gliad-U condition) was as high as prior to the GDF (before $202 \mathrm{U} / \mathrm{mL}$; after $182 \mathrm{U} / \mathrm{mL}$ ). The remaining 7 samples had a 3- to 33-fold reduction. Finally, for the tTGGliad condition, 35 out of 36 samples were positive before GFD. After the introduction of the GFD, 17 out of 35 samples became negative. Although the remaining 19 samples were positive, its concentration was 3 to 29 times lower than before introducing the GFD, except for two samples that did not vary, one of them being the same as in the two other conditions.

The same comparison before and after introducing a GFD was made using IgG anti-tTG levels (Fig. 3) with the group of $36 \mathrm{CD}$ patients. For the tTG-Gliad-U condition, only 28 out of 36 samples were positive before introducing

Table 1. Detection of IgA Anti-tTG Antibodies in 165 Non-Celiac Patients Using the Three ELISA Variants

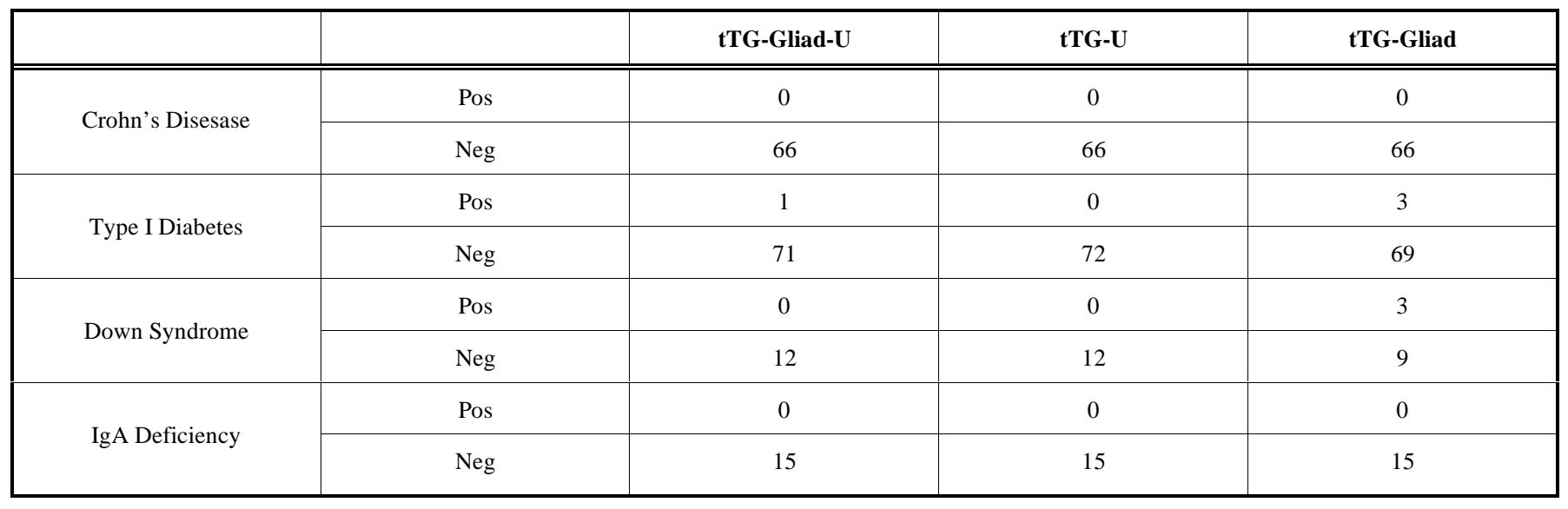



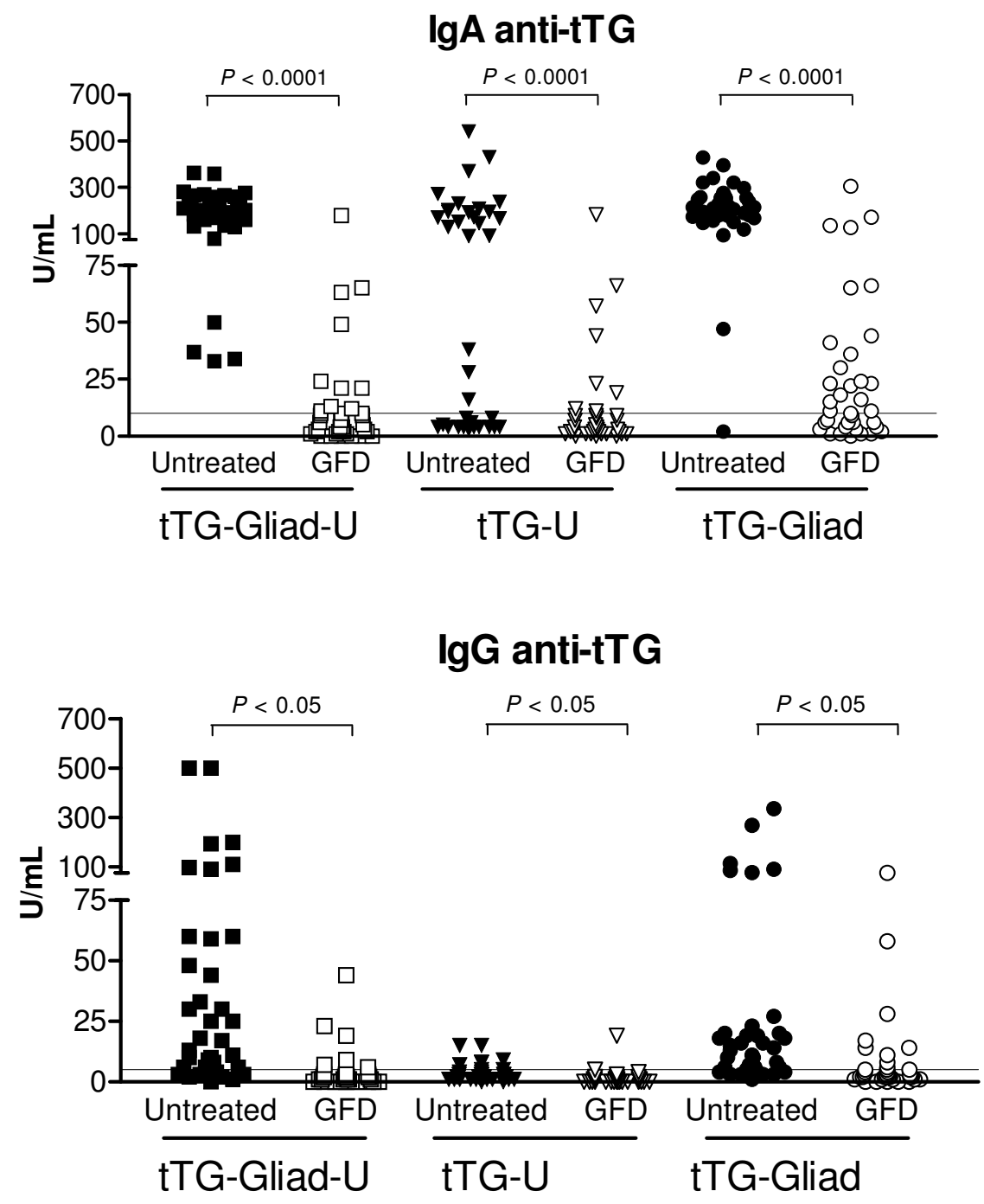

Fig. (3). Gluten-free diet follow-up. A gluten-free diet (GFD) was introduced in 36 celiac patients. Measurements of anti-tTG were done in samples obtained before and after the introduction of the GFD, using the three ELISA developed. Dotted line indicates the cut-off level in each figure. $P$ values are calculated for each test showing a statistically significant decrease in all of them.

the GFD. During the follow-up, 21 out of 28 positive samples became negative. The concentration of 6 samples suffered a reduction between 2-fold and 22-fold, whereas one sample did not vary. For the tTG-U condition, only 6 out of 36 samples were positive before introducing the GFD. After the retrieval of gliadin from the diet, 5 out of 6 samples became negative and the same sample as in the tTG-U condition remained low positive. Finally, for the tTG-Gliad condition, 27 out of 36 samples were positive before GFD. After the introduction of the GFD, 18 out of 27 positive samples became negative. 9 samples remained positive, but with con- centrations 1.3 to 9 times lower. In two cases concentrations were higher than before introducing the GFD.

\section{IgG Anti-tTg Levels in Celiac Patients with Selective IgA Deficiency}

A group of 14 celiac patients was chosen to evaluate the diagnostic characteristics in selective $\operatorname{IgA}$ deficiency. A group of $15 \mathrm{IgA}$-deficient non-celiac patients was used as disease control. All samples were negative for the IgA antitTG determination. Using the tTG-Gliadin-U condition with the IgG conjugate, 9 out of 14 celiac patients and none of the

Table 2. IgG Anti-tTG Antibodies in Celiac and Non-Celiac IgA Deficient Subjects

\begin{tabular}{|c|c|c|c|}
\hline \multirow{2}{*}{ Clinical samples } & \multirow{2}{*}{ IgG anti-tTG } & \multicolumn{2}{|c|}{ Pos } \\
\cline { 2 - 4 } & & 9 & 5 \\
\hline \hline IgA deficient celiac patients & 14 & 0 & 15 \\
\hline IgA deficient non celiac patients & 15 & 9 \\
\hline
\end{tabular}


Table 3. Effect of the Presence of Gliadin on the Determination of IgA Anti-tTG Antibodies

\begin{tabular}{|c|c|c|c|}
\hline & Gliad* & tTG & tTG-Gliad \\
\hline \hline Positive & 4 & 45 & 64 \\
\hline Negative & 63 & 22 & 3 \\
\hline
\end{tabular}

* Gliadin at the same low final concentration as in the mixture of tTG-Gliad.

control patients were positive (Table 2). Thus, sensitivity and specificity were 64.3 and $100 \%$, respectively.

From these results, the variant using microplates coated with tTG and gliadin, and urea in the sample buffer was chosen as the best condition for both, IgA and IgG anti-tTG assays. The activation of tTG by gliadin provides a higher rate of positive $\mathrm{CD}$ sera. In order to demonstrate that this increase was not due to the binding of potential antibodies to gliadin, we tested the same sera in microplates coated with the same amount of gliadin as in the mixture without tTG (Table 3). In such microplates, only 4 out of 67 sera are positive. On the other hand, as shown in Table 1, only 1 false positive out of 165 expected negatives was found when urea was present in the sample diluent, whereas 6 false positives were found when urea was not present.

\section{Technical Performance of the IgA or IgG tTG-Gliad-U ELISA}

Since the tTG-Gliad-U ELISA showed the best clinical performance, we next assessed its technical performance.

\section{Linearity}

Sera with high concentrations of $\operatorname{IgA}$ or IgG anti-tTG antibodies were diluted with sample diluent buffer to asses assay linearity in dilution experiments. Ratios between observed and expected values for each dilution were close to $100 \%$, hence the assay showed linearity over the full measuring interval (Fig. 4).

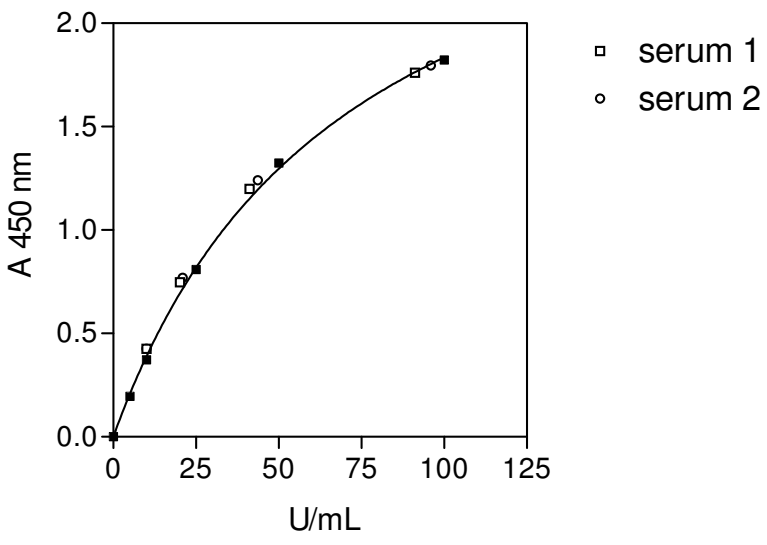

Fig. (4). Assay linearity in dilution experiments with sera with high concentrations of IgA anti-tTG antibodies diluted with sample diluent buffer. Concentrations of the serial dilutions of two sera are given respect to the calibration curve.

\section{Imprecision}

Intra-assay imprecision was estimated by 25 replicated measurements in a single run for each conjugate. Inter-assay imprecision was calculated from the results of 5 different runs with 5 determinations of each sample and each conjugate. Samples containing low to high IgA or IgG anti-tissue transglutaminase antibodies concentration were used (Table 4).

\section{Quantification Limit}

Estimated by performing 25 replicated measurements of the blank using each conjugate. The quantification limit was calculated as $10 \times \mathrm{x}$, where $s$ is the standard deviation of the blank repeated measurements, and it was found to be 5.2 $\mathrm{U} / \mathrm{mL}$ for IgA anti-tTG and $0.4 \mathrm{U} / \mathrm{mL}$ for IgG anti-tTG.

\section{Analytical Sensitivity}

The result is expressed as the slope of the calibration function within the interval comprised between 5 and 10 $\mathrm{U} / \mathrm{mL}$ and it is expressed as absorbance units per concentration units. It was found to be 0.022 for IgA anti-tTG and 0.054 for IgG anti-tTG.

\section{Interferences}

Possible interfering substances like hemoglobin, triglycerides, bilirubin and rheumatoid factor were investigated. We evaluated the interference by adding increasing concentrations of the interfering agent to 3 human sera with none, medium and high IgA or IgG anti-tTG concentrations. There were no interferences in the sera due to hemoglobin (up to $1000 \mathrm{mg} / \mathrm{dL}$ ), triglycerides (up to $3 \mathrm{~g} / \mathrm{dL}$ ), bilirubin (up to 40 $\mathrm{mg} / \mathrm{dL}$ ) and rheumatoid factor (up to $300 \mathrm{IU} / \mathrm{mL}$ ), for both conjugate specificities.

\section{Microplate Stability}

A highly positive celiac serum was tested on microplates to study the stability of the microplates under stressing conditions. The results obtained with sealed microplates incubated 7 days at $37^{\circ} \mathrm{C}$ showed a non-significant decrease of 2 $-4 \%$, as compared with microplates kept at $2-10^{\circ} \mathrm{C}$. Microplates that had not been treated with the blocking solution described in Materials and Methodology showed, instead, a significant decrease of 20 to $35 \%$ in absorbance.

\section{DISCUSSION}

The aim of the present study was to assess the performance of three anti-tTG ELISA tests variants using IgA and IgG conjugates, combining the presence or absence of calcium and gliadin and also the presence of urea in the sample diluent, and to evaluate the possible use of IgG anti-tTG in CD diagnosis.

The coating conditions compared differ on the presence or not of gliadin, but $5 \mathrm{mM}$ calcium was always present. Presence of calcium in the coating buffer has been controversial. Some authors claim that its presence improves the sensitivity of the ELISA because the antibodies of celiac 
Table 4. Imprecision. CV: Coeficient Variation

\begin{tabular}{|c|c|c|c|c|c|c|c|}
\hline \multicolumn{4}{|c|}{$\begin{array}{c}\text { tTG-IgA } \\
\text { Repeatability (intra) }\end{array}$} & \multicolumn{3}{|c|}{ Reproducibility (inter) } & \multirow[b]{2}{*}{$N$} \\
\hline & $U / m L$ & $C V \%$ & $n$ & & $U / m L$ & $C V \%$ & \\
\hline sample 1 & 10.1 & 7.8 & 25 & sample 1 & 10.1 & 10.3 & 25 \\
\hline sample 2 & 36.1 & 6.9 & 25 & sample 2 & 36.1 & 10.0 & 25 \\
\hline & $U / m L$ & $\mathrm{CV} \%$ & $n$ & & $U / m L$ & $\mathrm{CV} \%$ & $n$ \\
\hline sample 1 & 13.4 & 3.8 & 24 & sample 1 & 29.1 & 7.6 & 25 \\
\hline sample 2 & 41.0 & 3.2 & 24 & sample 2 & 49.9 & 6.8 & 25 \\
\hline
\end{tabular}

patients react more strongly with the calcium-activated form of tTG due to conformational changes of the enzyme molecule $[6,7,8,9,10]$ but other authors find that calcium is not essential $[11,12]$. We have chosen to add $5 \mathrm{mM}$ of calcium in the coating buffer although having only a mild improvement on the analytical sensitivity in preliminary experiments (data not shown).

Gliadin, in the presence of calcium, increases sensitivity of IgA anti-tTG antibodies up to $95.5 \%$, compared to $65.0 \%$ without gliadin (Table 1, Fig. 2). This increase was not due to the binding of potential antibodies to gliadin because in microplates coated with gliadin alone (in the same amount as in the mixture with tTG) only $6.0 \%$ of the sera were positive (Table 3). Thus, tTG alone is not sufficient to be recognized by the antibodies and only when tTG and gliadin are present total recognition occurs. Activation of tTG by calcium and gliadin may play a role for a complete expression of tTG autoepitopes during the coating process [13].

Together with the coating conditions, we have also compared two sample diluents, in the presence or absence of urea. The incorporation of urea to the sample diluent reduces the non-specific binding of immunoglobulins to the antigen (and/or the solid phase support material used in the assay) because there is an increase in the stringency of the antibody-antigen interaction, excluding or, at least, reducing the number of false positives due to low-avidity complexes.

The best results are, therefore, obtained using microplates with tTG activated with calcium and gliadin, and with urea in the sample diluent. Diagnostic sensitivity and specificity for IgA anti-tTG from CD patients with total or subtotal villous atrophy, $(95.5 \%$ and $99.4 \%$, respectively) are comparable to those described in the literature $[1,14]$.

On the other hand, our results with the IgG anti-tTG show a high specificity (97\%) but a lower sensitivity (79\%). Reported sensitivity values for IgG anti-tTG antibodies range from 21 to $98 \%[11,15-17]$ in studies with CD patients without IgA deficiency. Such sensitivity values are equal or lower than those obtained for IgA anti-tTG antibodies, therefore detection of IgG anti-tTG does not seem to offer advantages in the diagnosis of celiac disease. Differ- ences between $\operatorname{IgA}$ and IgG anti-tTG antibodies may be due to the different epitopes recognized by each isotype [18].

Removal of gluten from the diet results in an improvement of clinical symptoms together with a decrease in antibody titers [19-21]. Our results indicate that IgA anti-tTG antibodies are reduced to normal or significantly lower levels in those patients receiving a GFD. However, further studies should be carried out to gain knowledge of the influence of the patients age or the GFD duration on the reduction of the antibodies titers. In the case of the tTG-Gliad-U condition, we observed only 1 case with persistent high IgA anti-tTG level. These results agree with several reports in which determination of $\operatorname{IgA}$ anti-tTG is found to be useful in the follow-up of $\mathrm{CD}$ in children $[15,20]$ and adults [22, 23], although some authors state that $\operatorname{IgA}$ anti-tTG assay is not better than anti-gliadin antibodies (AGA) or anti-endomysium antibodies (EmA) in assessing histologic recovery after GFD and in assessing dietary transgression [24, 25].

It has been proposed that the detection of IgG anti-tTG antibodies can be a useful marker for $\mathrm{CD}$ in selective $\operatorname{Ig} \mathrm{A}$ deficiency $[14,26]$ or in the follow-up of the disease after treatment $[19,20,27]$. In a study comparing 9 commercial IgG anti-tTG ELISA in CD patients with selective IgA deficiency, sensitivity values ranged from $75 \%$ to $95 \%$ [27]. Sensitivity obtained in our studies for the tTG-Gliadin-U condition $(79 \%)$ is within the range.

Concerning the follow-up of CD with normal concentrations of $\mathrm{IgA}$ it has been reported that IgG anti-tTG antibodies decrease in a similar rate than IgA anti-tTG antibodies after gluten removal [15]. Our results with $\operatorname{IgG}$ are limited to those samples found to be positive before GFD, but we observe a decrease similar to $\operatorname{IgA}$. In IgA-deficient patients, usefulness of IgG anti-tTG antibodies for diagnosis and follow-up of CD is still not clear because assays for detection of IgG anti-tTG antibodies perform not as good as the $\mathrm{IgG}$ AGA assays [14].

In summary, we have shown that to obtain the best results on the clinical performance of microplates coated with human tTG, calcium and gliadin are required, together with the use of a stringent sample buffer. Under these conditions, 
the human recombinant tTG-based ELISA for IgA anti-tTG antibodies is sensitive and specific, and a reliable tool to ensure the compliance with diet of CD patients.

\section{AKNOWLEDGEMENTS}

We would like to thank Ana Belén Polonio and Sabina Dueñas for their technical assistance throughout this study.

\section{REFERENCES}

[1] Green PHR, Jabri B. Celiac disease. Annu Rev Med 2006; 57: $207-$ 21.

[2] Sakly W, Thomas V, Quash G, El Alaoui S. A role for tissue transglutaminase in $\alpha$-gliadin peptide cytotoxicity. Clin Exp Immunol 2006; 146(3): 550-8

[3] Folk JE, Finlayson JS. The Ne-( $\gamma$-glutamyl) lysine crosslink and the catalytic role of transglutaminases. Adv Protein Chem 1977; 31:1133.

[4] Sollid M. Molecular basis of coeliac disease. Annu Rev Immunol 2000; 18:53-81.

[5] Ciccocioppo R, Di Sabatino A, Ara C, Di Sabatino A, Mengheri E, Corazza GR. Gliadin and tissue transglutaminase complexes in normal and coeliac duodenal mucosa. Clin Exp Immunol 2003; 134(3): 516-24

[6] Dieterich W, Laag E, Schöpper H, et al. Autoantibodies to tissue transglutaminase as predictors of celiac disease. Gastroenterology 1998; 115: 1317-21.

[7] Sulkanen S, Halttunen T, Laurila K, et al. Tissue transglutaminase autoantibody enzyme-linked immunosorbent assay in detecting celiac disease. Gastroenterology 1998; 115: 1322-8.

[8] Sárdy M, Odenthal U, Kárpáti S, Paulsson M, Smyth N. Recombinant human tissue transglutaminase ELISA for the diagnosis of gluten-sensitive enteropathy. Clin Chem 1999; 45(12): 2142-9.

[9] Agardh D, Roth B, Lernmark $\AA$, Stenberg P. Calcium activation of tissue transglutaminase in radioligand binding and enzyme-linked autoantobody immunoassays in childhood celiac disease. Clin Chim Acta 2005; 358(1-2): 95-103.

[10] Roth EB, Sjöberg K, Stenberg P. Biochemical and immunopathological aspects of tissue transglutaminase in coeliac disease. Autoimmunity 2003; 36(4): 221-6.

[11] Troncone R, Maurano F, Rossi M, et al. $\mathrm{IgA}$ antibodies to tissue transglutaminase: An effective diagnostic test for celiac disease. J Pediatr 1999; 134: 166-71.

[12] Dahele AVM, Aldhous K, Humphreys K, Ghosh S. Serum IgA tissue transglutaminase antibodies in coeliac disease and other gastrointestinal diseases. QJM 2001; 94:195-205.

[13] Maassen A, Conrad K, Porstmann T. Diagnosis of celiac disease by ELISA: the importance of the formation of neoepitopes of tissue transglutaminase (tTG) versus the human or guinea pig origin. In: Conrad K, Humbel RL, Meurer M, Shoenfeld Y, Tan EM, Eds. Autoantigens and autoantibodies: diagnostic tools and clues to un- derstanding autoimmunity. PABST Science Publishers 2000: pp. 552-553.

[14] Van Meensel B, Hiele M, Hoffman I, Vermeire S, Rutgeerts P, Geboes K, Bossuyt X. Diagnostic accuracy of ten secondgeneration (human) tissue transglutaminase antibody assays in celiac disease. Clin Chem 2004; 50(11): 2125-35.

[15] Martín-Pagola A, Ortiz-Paranza L, Bilbao JR, et al. Two-year follow-up of anti-transglutaminase autoantibodies among celiac children on gluten-free diet: Comparison of IgG and IgA. Autoimmunity 2007; 40(2): 117-21.

[16] Bazzigaluppi E, Lampasona V, Barera G, et al. Comparison of tissue transglutaminase-spcific antibody assays with established antibody measurements for coeliac disease. J Autoimmun 1999; 12: 51-6.

[17] Seissler J, Boms S, Wohlrab U, et al. Antibodies to human recombinant tissue transglutaminase measured by radioligand assay: evidence for high diagnostic sensitivity for celiac disease. Horm Metab Res 1999; 31: 375-9.

[18] Byrne G, Ryan F, Jackson J, Feighery C, Kelly J. Mutagenesis of the catalytic triad of tissue transglutaminase abrogates coeliac disease serum IgA autoantibody binding. Gut 2007; 56(3): 336-41

[19] Agardh D, Borulf S, Lernmark $\AA$, Ivarsson SA. Tissue transglutaminase immunoglobulin isotypes in children with untreated and treated celiac disease. J Pediatr Gastroenterol Nutr 2003; 36:77-82.

[20] Hansson T, Dahlbom I, Rogberg S, et al. Recombinant human tissue transglutaminase for diagnosis and follow-up of childhood coeliac disease. Pediatr Res 2002; 51(6):700-705.

[21] Cataldo F, Marino V, Ventura A, Bottaro G, Corazza GR. Prevalence and clinical features of selective immunoglobulin A deficiency in coeliac disease: an Italian multicentre study. Gut 1998; 42: 362-5.

[22] Midhagen G, Åberg AK, Olcén P, et al. Antibody levels in adult patients with coeliac disease during gluten-free diet: a rapid initial decrease of clinical importance. J Intern Med 2004; 256(6): 519-24.

[23] Kaukinen K, Sulkanen S, Mäki M, Collin P. IgA-class transglutaminase antibodies in evaluating the efficacy of gluten-free diet in coeliac disease. Eur J Gastrenterol Hepatol 2002; 14(3): 311-5.

[24] Tursi A, Brandimarte G, Giorgetti GM. Lack of usefulness of antitransglutaminase antibodies in assessing histologic recovery aftergluten-free diet in celiac disease. J Clin Gastroenterol 2003; 37(5): 387-91.

[25] Hopper AD, Hadjivassiliou M, Hurlstone DP, et al. What is the role of serologic testing in celiac disease? A prospective, biopsyconfirmed study with economic analysis. Clin Gastroenterol Hepatol 2008; 6(3): 314-20.

[26] Korponay-Szabó IR, Dahlbom I, Laurila K, et al. Elevation of IgG antibodies against tissue transglutaminase as a diagnostic tool for coeliac disease in selective IgA deficiency. Gut 2003; 52: 1567-71.

[27] Villalta D, Alessio MG, Tampoia M, et al. Testing for IgG class antibodies in celiac disease patients with selective $\operatorname{IgA}$ deficiency. A comparison of the diagnostic accuracy of $9 \mathrm{IgG}$ anti-tissue transglutaminase, $1 \mathrm{IgG}$ anti-gliadin and 1 anti-deaminated gliadin peptide antibody assays. Clin Chim Acta 2007; 382(1-2): 95-9. 